\title{
Preservation of ageing classes in deterioration models with independent increments
}

\author{
Carmen Sangüesa', Francisco German Badía ${ }^{2}$ and Ji Hwan Cha ${ }^{3 *}$
}

\section{"Correspondence:}

jhcha@ewha.ac.kr

${ }^{3}$ Department of Statistics, Ewha

Womans University, Seoul, 120-750,

Korea

Full list of author information is

available at the end of the article

\begin{abstract}
In the present paper we consider ageing properties in a deterioration model in which the stochastic process measuring deterioration is a process with independent increments. Preservation of increasing and decreasing failure rates, as well as decreasing reversed hazard rate, is considered. We also take into account the preservation of log-concave and log-convex densities. Our main results are based on technical results concerning preservation of log-concave and log-convex functions by positive linear operators, and they include the study of stochastic ordering properties among the random variables in the process.
\end{abstract}

MSC: 60G51; 60E15; 60K10; 26A51

Keywords: wear process; ageing class; stochastic order; log-concavity (log-convexity)

\section{Introduction}

Deterioration models belong to the topics of interest in reliability theory. They aim to describe how a mechanism deteriorates with age. A convenient way in modeling the uncertainty in time-dependent deterioration is by regarding it as a stochastic process. That is, deterioration in time of a device is described by a stochastic process $(X(t), t \geq 0)$, in which each $X(t)$ represents the degree of deterioration at an instant $t$. Gamma processes have been mainly considered to model degradation in time [1-3]. Also, the so-called shock models are appropriate if deterioration is caused due to external shocks occurring at certain instants in time (see, for instance, [4]). Although it seems more realistic to consider processes with non-negative increments in order to measure deterioration, Brownian motion has also been considered (geometric, with drift, or alone as an additional term measuring errors; see [5, 6], and the references therein). General Markovian processes have also been considered (see [7] and the references therein).

In a deterioration model, we assume that the system breaks down when the degree of deterioration attains a certain non-negative threshold $Y$ (which we will assume to be random and independent of the process $(X(t), t \geq 0))$, that is, the lifetime of the device is

$$
\rho=\inf \{t \geq 0: X(t) \geq Y\} .
$$

O2014 Sangüesa et al.; licensee Springer. This is an Open Access article distributed under the terms of the Creative Commons Attribution License (http://creativecommons.org/licenses/by/2.0), which permits unrestricted use, distribution, and reproduction in any medium, provided the original work is properly cited. 
As usual, for a random variable $Y$, we will denote by $F_{Y}$ the distribution function of this random variable and $\bar{F}_{Y}:=1-F_{Y}$ will stand for its survival function. Also, from now on, we shall use the words decreasing and increasing instead of non-increasing and non-decreasing, respectively. We will assume that $(X(t), t \geq 0)$ has increasing and rightcontinuous paths. In this case, as in [8, p.817], we can write

$$
\bar{F}_{\rho}(t):=P(\rho>t)=E\left[\bar{F}_{Y}(X(t))\right] ; \quad F_{\rho}(t):=P(\rho \leq t)=E\left[F_{Y}(X(t))\right], \quad t \geq 0 .
$$

The analytical form of previous functions is usually not easy to deal with, although several expressions are known for specific models (see [5] for the geometric Brownian motion, as well as for the gamma process when we have a fixed threshold). Then it seems natural to study under which conditions $\rho$ inherits from $Y$ the common reliability properties studied in the literature. In reliability theory, the principal ageing properties considered for a random variable involve the study of the log-concavity (positive ageing) or log-convexity (negative ageing) of a certain function (which usually is the distribution function, survival function or density function). For instance, if $\bar{F}_{Y}$ is log-concave, the random variable is said to be increasing failure rate (IFR), whereas if it is log-convex, we have the decreasing failure rate property (DFR). Moreover, $Y$ is said to be the decreasing reversed hazard rate (DRHR) if $F_{Y}$ is log-concave. We will recall the properties we are going to use in Definition 2.3, although a more detailed discussion can be found in [9, Ch. 2], for instance. In the context of deterioration models, preservation of common ageing properties for a fixed threshold has been studied, for instance, in [10] in a context of pure-jump processes. As far as we know, the problem of a random threshold was firstly considered by Esary et al. in a context of shock models [11] and by Abdel-Hameed in several papers [1, 8, 12]. In [1] a gamma wear process was considered, whereas in $[8,12]$ results are obtained for a pure-jump wear processes. See also [13] for a recent review. Our aim in this paper is to address this question for processes with independent increments, thus including Lévy processes. To this end, we use the representation given in (1) and apply techniques based on the preservation of log-convexity and log-concavity by positive linear operators (see $[14,15])$. These techniques involve the study of stochastic order properties of the random variables in the process. This approach is different from that used in [12] for pure-jump Lévy processes, which is based on the underlying Lévy measure of the process. Our results generalize previous ones for a compound Poisson process (see Remark 3.5), as well as for a gamma process (see Remark 3.8 and Remark 3.12). On the other hand, it is usual that preservation results of positive ageing properties (IFR, for instance) hold true under more restrictive assumptions than their analogous negative ageing properties (DFR). This can be seen in [12], Theorem 2.3, in which for the preservation of the IFR property the requirement is a log-concave density for the Lévy measure, whereas for the DFR property no assumption on this density is needed. Our approach also gives different conditions for the preservation of the IFR and DFR property (see Proposition 3.1(a) and Corollary 3.11, respectively, in Section 3). We also include preservation results concerning the DRHR property (Proposition 3.1(b)). This property is of recent interest and has not been dealt with in the afore-mentioned papers. It should be pointed out that the flexibility of our approach allows us to add deterministic trends without making an extra effort. This approach also allows us to prove stronger results, having to do with the log-convexity or log-concavity of the density function (Section 4). 


\section{Preliminaries}

As mentioned in the Introduction, the concept of log-concavity will play an important role in our results. We first recall this concept.

Definition 2.1 Let $I \subseteq \mathbb{R}$ be a convex set. A function $f: I \rightarrow[0, \infty]$ is said to be logconcave on $I$ if for all $x, y \in I$ and $0 \leq \alpha \leq 1$ it verifies that

$$
f(\alpha x+(1-\alpha) y) \geq f(x)^{\alpha} f(y)^{1-\alpha},
$$

or equivalently $\log f$ is concave (in the interval in which $f$ is strictly positive).

Remark 2.2 If the inequality in the previous definition is reversed, we obtain the dual concept of log-convexity.

Log-concavity is an important concept in reliability theory. Actually the principal ageing classes considered in the literature can be defined in terms of log-concavity or logconvexity (see, for instance, [9, Ch. 2]). We recall the definitions of the main ageing classes to be used along the paper.

Definition 2.3 Let $X$ be a non-negative random variable. $X$ is said to be:

(a) an increasing failure rate (IFR) if $\bar{F}_{X}$ is log-concave on $\mathbb{R}$;

(b) a decreasing reversed hazard rate (DRHR) if $F_{X}$ is log-concave on $\mathbb{R}$;

(c) log-concave if $X$ is absolutely continuous and its density $f_{X}$ is $\log$-concave on $(0, \infty)$.

If in parts (a) and (c) log-concavity is replaced by log-convexity we have the decreasing failure rate (DFR) and log-convex ageing classes, respectively. For the DFR property the log-convexity has to be restricted to $[0, \infty)$.

Remark 2.4 It is interesting to point out that $X$ log-concave $\Rightarrow X$ IFR and DRHR, and that $X$ log-convex $\Rightarrow X$ DFR $\Rightarrow X$ DRHR (see $[9, \mathrm{p} .181]$ ).

It is reasonable to assume that, in a deterioration process, each $X(t)$ is non-negative, and that the degree of deterioration increases with $t$ (in a certain stochastic order). In the next definition we recall the different stochastic orders we are going to use in our deterioration models. For a more detailed discussion, see [16, 17], for instance.

Definition 2.5 Let $X$ and $Y$ be two random variables. $X$ is said to be smaller than $Y$ in

(i) the usual stochastic order (written as $X \leq_{\text {st }} Y$ ) if $\bar{F}_{X}(x) \leq \bar{F}_{Y}(x)$, for all $x \in \mathbb{R}$;

(ii) the hazard rate order $(X \leq \mathrm{hr} Y)$ if $\bar{F}_{Y}(t) / \bar{F}_{X}(t)$ is increasing in $t$;

(iii) the reversed hazard rate order $\left(X \leq_{\mathrm{rh}} Y\right)$ if $F_{Y}(t) / F_{X}(t)$ is increasing in $t$;

(iv) the likelihood ratio order $\left(X \leq_{\operatorname{lr}} Y\right)$ if $X$ and $Y$ are absolutely continuous with respect to some dominating measure $\mu$, with respective densities $f_{X}$ and $f_{Y}$ such that $f_{Y}(t) / f_{X}(t)$ is increasing in $t$.

Remark 2.6 The relations among the previous stochastic orders are as follows (see [16, p.61]):

- $X \leq_{\mathrm{lr}} Y \Rightarrow X \leq_{\mathrm{hr}} Y$ and $X \leq_{\mathrm{rh}} Y$;

- Either $X \leq_{\mathrm{hr}} Y$ or $X \leq_{\mathrm{rh}} Y \Rightarrow X \leq_{\mathrm{st}} Y$. 
For a given process $(X(t), t \geq 0)$, we will use the notation $X(t) \uparrow$. to indicate that $X(t)$ is increasing in the stochastic order, for all $t \geq 0$ (and $X(t) \downarrow$. if it is decreasing). From now on, we will use the notation $X={ }_{\text {st }} Y$ to indicate that two random variables $X$ and $Y$ have the same distribution. In next definition we will describe the properties we will assume for the process $(X(t), t \geq 0)$, which are slightly more general than the ones defining a non-negative Lévy process.

Definition 2.7 Let $(X(t), t \geq 0)$ be a stochastic process. We will say that this process belongs to the class $\mathcal{I P} \mathcal{I} \mathcal{I}$ (independent positive increasing increments) if it verifies:

1. $0 \leq X(s) \leq X(t)$ a.s., for $0 \leq s<t$;

2. the process has independent increments, that is: given $0 \leq t_{0}<t_{1}<\cdots<t_{n}$, the random variables $X\left(t_{0}\right), X\left(t_{1}\right)-X\left(t_{0}\right), \ldots, X\left(t_{n}\right)-X\left(t_{n-1}\right)$ are independent;

3. the increments of the process satisfy

$$
X(t+h)-X(t) \uparrow \text { st } \quad \text { in } t \text { for any fixed } h>0 \text {; }
$$

4. $(X(t), t \geq 0)$ is continuous in probability, that is, $\lim _{s \rightarrow t} P(|X(t)-X(s)|>\epsilon)=0$, for all $\epsilon>0$.

If condition 3 is replaced by $X(t+h)-X(t) \downarrow_{\text {st }}$ in $t$, we will say that the process belongs to the $\mathcal{I P D} \mathcal{I}$ (independent positive decreasing increments), whereas if the process belongs to $\mathcal{I P} \mathcal{D} \mathcal{I} \cap \mathcal{I} \mathcal{P} \mathcal{I}$, that is,

$$
X(u+h)-X(u)=_{\mathrm{st}} X(v+h)-X(v), \quad u, v \geq 0, h>0
$$

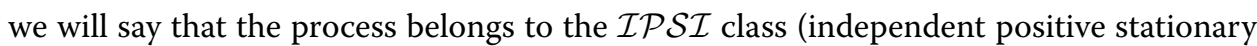
increments).

Particular examples of processes satisfying the above properties, which will be used along the paper, are the following:

- The standard Poisson process $(N(t), t \geq 0)$, which is a process in the $\mathcal{I} \mathcal{P} \mathcal{S}$ class such that $N(0)=0$, and such that, for each $t>0, N(t)$ has Poisson distribution of mean $t$ (see [18, p.15]).

- The standard gamma process $(S(t), t \geq 0)$, which is a process in the $\mathcal{I P S \mathcal { I }}$ class such that $S(0)=0$, and such that, for each $t>0, S(t)$ has gamma density $f(x):=\Gamma(t)^{-1} x^{t-1} e^{-x}, x>0$ (see, for instance, [3, p.6]).

Remark 2.8 Note that the processes considered in Definition 2.7 admit always a representation with right-continuous paths $[18$, p.63], so that the expressions given in (1) hold true. Note also that if $X(0)=0$, the $\mathcal{I} \mathcal{P} \mathcal{S I}$ class coincides with Lévy processes with non-negative increments (or subordinators [18, p.137]). In fact, the processes we are going to deal with mainly (compound Poisson process, $c f$. [18, p.18] and gamma process) belong to this class. Moreover, it is readily seen (we include the proof of this fact in Lemma 3.3) that for a given process $(X(t), t \geq 0)$ in the $\mathcal{I P} \mathcal{S I}$ class, the time-transformed process $\left(X\left(a_{2}(t)\right), t \geq 0\right)$, with $a_{2}$ being an increasing and convex function, belongs to the $\mathcal{I P} \mathcal{I} \mathcal{I}$ class, whereas if $a_{2}$ is increasing and concave, the process belongs to the $\mathcal{I P} \mathcal{D} \mathcal{I}$ class. We will use this fact in order to obtain results concerning non-homogeneous Poisson processes (Proposition 3.4) 
and non-homogeneous gamma processes (Proposition 4.4), which are time-transformed versions of the standard Poisson and gamma processes, respectively.

Finally, we state a technical result, which can be found in [15] and will play an important role in our proofs.

Theorem 2.9 ([15], Thm. 3.8) Let $(X(t), t \geq 0)$ be a stochastic process satisfying condition 1 in Definition 2.7. Let $f$ be the set of measurable functions $f:[0, \infty) \rightarrow[0, \infty)$ such that $E f[X(t)]<\infty, t \geq 0$. Let $T$ be an operator defined as

$$
T f(t)=E f[X(t)], \quad t \geq 0 .
$$

Assume that the following assumptions are verified:

1. $f$ is log-concave;

2. Tf is continuous on $(0, \infty)$.

(a) Further assume that $f$ is decreasing. If $(X(t), t \geq 0)$ is in the class $\mathcal{I} \mathcal{I} \mathcal{I}$ and $X(t) \uparrow_{\mathrm{rh}}$, then $T f$ is a log-concave and decreasing function on $(0, \infty)$.

(b) Further assume that $f$ is increasing. If $(X(t), t \geq 0)$ is in the class $\mathcal{I P D I}$ and $X(t) \uparrow \mathrm{hr}$ then $T f$ is a log-concave and increasing function on $(0, \infty)$.

(c) If $(X(t), t \geq 0)$ is in the class $\mathcal{I P S I}$ and $X(t) \uparrow_{\mathrm{Ir}}$, then Tf is a log-concave function on $(0, \infty)$

Remark 2.10 If $T f$ is continuous at the origin we can extend the log-concavity property to the interval $[0, \infty)$, as (2) at 0 as endpoint can be deduced by taking the limit as $x \downarrow 0$.

Remark 2.11 In [15], Thm. 3.8(c) there is an additional condition. If, for $f:[0, \infty) \rightarrow$ $[0, \infty)$, we call $J:=\{x \geq 0 \mid f(x)>0\}$ (which is an interval if $f$ is log-concave), the additional condition was that the set $J^{*}:=\{t>0 \mid P(X(t) \in J)>0\}$ had to be an interval. But the previous condition is always verified if $X(t) \uparrow_{\mathrm{lr}}$, so that it does not need to be checked. In the next lemma we give the proof of this fact.

Lemma 2.12 Let $(X(t), t \geq 0)$ be a stochastic process such that $X(t) \uparrow \uparrow_{\mathrm{lr}}$ and let $J \subseteq \mathbb{R}$ be an interval. Then $J^{*}:=\{t \geq 0 \mid P(X(t) \in J)>0\}$ is an interval.

Proof To show the assertion we will prove that

$$
P\left(X\left(t_{1}\right) \in J\right) P\left(X\left(t_{2}\right) \in J\right)>0, \quad 0 \leq t_{1}<t_{2} \quad \Rightarrow \quad P(X(t) \in J)>0 \quad \text { for all } t_{1}<t<t_{2} .
$$

We will use a reduction to absurd argument. Assume that $P\left(X\left(t_{1}\right) \in J\right) P\left(X\left(t_{2}\right) \in J\right)>0$, but $P(X(t) \in J)=0$, for some $t_{1}<t<t_{2}$. We will use the property ( $c f$. [17, p.42]) that if $X_{1} \leq_{\operatorname{lr}} X_{2}$, and $A$ and $B$ are Borel sets in $[0, \infty)$, then

$$
P\left(X_{1} \in B\right) P\left(X_{2} \in A\right) \leq P\left(X_{1} \in A\right) P\left(X_{2} \in B\right), \quad \text { if } A \leq B,
$$

where $A \leq B$ means that $x \leq y$ for all $x \in A$ and $y \in B$. We will denote by $J_{+}^{c}$ the set of real numbers above $J$ and by $J_{-}^{c}$ the set of real numbers below $J$. Using our assumption, and 
applying (4) with $X_{1}:=X\left(t_{1}\right), X_{2}:=X(t), A=J_{-}^{c}$ and $B=J$, we obtain

$$
P\left(X\left(t_{1}\right) \in J\right) P\left(X(t) \in J_{-}^{c}\right)=0 \quad \Rightarrow \quad P\left(X(t) \in J_{-}^{c}\right)=0,
$$

whereas (4) with $X_{1}:=X(t), X_{2}:=X\left(t_{2}\right), A=J$ and $B=J_{+}^{c}$ implies that

$$
P\left(X(t) \in J_{+}^{c}\right) P\left(X\left(t_{2}\right) \in J\right)=0 \quad \Rightarrow \quad P\left(X(t) \in J_{+}^{c}\right)=0 .
$$

Then (5) and (6) are contradictory with the fact that $P(X(t) \in J)=0$, and the conclusion follows.

\section{Preservation of IFR, DRHR, and DFR classes for wear processes with independent increments}

In order to ensure the continuity condition in Theorem 2.9, we will assume that $Y$ does not have common points with positive mass with $(X(t), t>0)$. This can be stated as follows:

$$
P(X(t) \in D)=0 \text { for all } t>0 \text {, where } D:=\{x \geq 0 \mid P(Y=x)>0\} .
$$

Our first results, concerning to the classes IFR and DRHR, are based on the following.

Proposition 3.1 Let $(X(t), t \geq 0)$ be a wear process with random threshold $Y$ satisfying condition (7). Let $\rho$ be the failure time of the device. Then we have:

(a) If $(X(t), t \geq 0)$ is in the IPII class with $X(0)=0, X(t) \uparrow_{\mathrm{rh}}$ and $Y$ is IFR, then $\rho$ is IFR.

(b) If $(X(t), t \geq 0)$ is in the IPDI class, $X(t) \uparrow \mathrm{hr}$ and $Y$ is DRHR, then $\rho$ is DRHR.

Proof Condition (7) and (1) ensures us that $F_{\rho}$ and $\bar{F}_{\rho}$ are continuous functions on $(0, \infty)$ [15, Lem. 2.5]. The fact that $F_{\rho}$ and $\bar{F}_{\rho}$ are right-continuous and condition 4 in Definition 2.7 allow us to extend the continuity to $[0, \infty)$.

To show part (a), the IFR condition for $Y$ means that $\bar{F}_{Y}$ is log-concave. Thus, by (1), Theorem 2.9(a) and Remark 2.10 we find that $\bar{F}_{\rho}$ is log-concave on $[0, \infty)$. To extend this property to $\mathbb{R}$, note that an IFR distribution cannot have positive mass at 0 (see [9, p.104]). The fact that $X(0)=0$ guarantees this property for $\rho$, as by $(1) \bar{F}_{\rho}(0)=\bar{F}_{Y}(0)=1$. Thus, using this property, the log-concavity property for $\bar{F}_{\rho}$ is extended to $\mathbb{R}$, thus showing part (a).

For part (b), the DRHR condition for $Y$ means that $F_{Y}$ is log-concave, and by (1), Theorem 2.9(b), and Remark 2.10 we find that $F_{\rho}$ is log-concave on $[0, \infty)$. As $F_{\rho}(t)=0, t<0$, the log-concavity property is trivially extended to $\mathbb{R}$.

Remark 3.2 Recall that $X \leq_{\mathrm{lr}} Y$ implies both $X \leq_{\mathrm{hr}} Y$ and $X \leq_{\mathrm{rh}} Y$. So that $X(t) \uparrow_{\mathrm{lr}}$, together with $(X(t), t \geq 0)$ in the class $\mathcal{I P S I}$ and $X(0)=0$ are sufficient conditions for the preservation of both the IFR and the DRHR property.

In our next results we provide conditions to check log-concavity when we modify a process $(X(t), t \geq 0)$ in the $\mathcal{I P S I}$ class by adding a deterministic trend and applying a time transformation. To this end, let $a_{i}:[0, \infty) \rightarrow[0, \infty), i=1,2$ be two increasing and continuous functions. Consider the modified process:

$$
X^{*}(t)=a_{1}(t)+X\left(a_{2}(t)\right), \quad t \geq 0 .
$$


First of all we have the following.

Lemma 3.3 Let $(X(t), t \geq 0)$ be a process in the $\mathcal{I P S I}$ class. Consider $\left(X^{*}(t), t \geq 0\right)$ as defined above. Then we have:

(a) If $a_{1}$ and $a_{2}$ are increasing and convex functions, then $\left(X^{*}(t), t \geq 0\right)$ is in the $\mathcal{I P I I}$ class.

(b) If $a_{1}$ and $a_{2}$ are increasing and concave functions, then $\left(X^{*}(t), t \geq 0\right)$ is in the $\mathcal{I P D I}$ class.

Proof To show part (a), note that conditions 1,2, and 4 in Definition 2.7 are clear by the corresponding ones for $(X(t), t \geq 0)$, taking into account that $a_{1}$ and $a_{2}$ are increasing and continuous. To verify condition 3 , call

$$
i_{h}(t):=a_{1}(t+h)-a_{1}(t), \quad I_{h}(t):=X\left(a_{2}(t+h)\right)-X\left(a_{2}(t)\right), \quad t \geq 0, h>0 .
$$

We have

$$
X^{*}(t+h)-X^{*}(t)=i_{h}(t)+I_{h}(t) .
$$

Our aim is to show that

$$
i_{h}(s) \leq i_{h}(t), \quad I_{h}(s) \leq_{\mathrm{st}} I_{h}(t), \quad 0 \leq s<t, h>0 .
$$

The first inequality is obvious, as $a_{1}$ is convex. For the second inequality, call $h(t)=a_{2}(t+$ $h)-a_{2}(t), t \geq 0$. As $a_{2}$ is convex, we see that $h(\cdot)$ is increasing. On the other hand if we use (3) for the $(X(t), t \geq 0)$ process with $u=a_{2}(s), v=a_{2}(t)$ and $h=h(s)$, we obtain

$$
\begin{aligned}
I_{h}(s) & =X\left(a_{2}(s)+h(s)\right)-X\left(a_{2}(s)\right)==_{\text {st }} X\left(a_{2}(t)+h(s)\right)-X\left(a_{2}(t)\right) \\
& \leq_{\text {st }} X\left(a_{2}(t)+h(t)\right)-X\left(a_{2}(t)\right)=I_{h}(t),
\end{aligned}
$$

in which, in the last inequality, we have used that $h$ is increasing and condition 1 for the $(X(t), t \geq 0)$ process (non-negative increments a.e.). This proves the second inequality in (11). Thus, as the stochastic order is preserved under addition of a non-negative constant, we deduce from (10) and (11) that

$$
X^{*}(s+h)-X^{*}(s)=i_{h}(s)+I_{h}(s) \leq_{\mathrm{st}} i_{h}(t)+I_{h}(t)=X^{*}(t+h)-X^{*}(t), \quad 0 \leq s<t,
$$

so that condition 3 is proved, thus concluding part (a).

Part (b) is proved taking into account that the inequalities in (11) are reversed if $a_{i}, i=1,2$ are concave functions.

Using the two previous results we have the following result concerning a non-homogeneous compound Poisson process.

Proposition 3.4 Let $X^{*}(t)$ be a non-homogeneous compound Poisson wear process, that is,

$$
X^{*}(t)=\sum_{i=1}^{N^{*}(t)} X_{i},
$$


in which $\left(N^{*}(t), t \geq 0\right)$ is a non-homogeneous Poisson process with mean function $\left(a_{2}(t):=\right.$ $\left.E\left[N^{*}(t)\right], t \geq 0\right)$ and $\left(X_{n}\right)_{n=1,2, \ldots}$ is a sequence of independent non-negative identically distributed random variables, independent of the process. Assume that $Y$, the random threshold and $\left(X^{*}(t), t \geq 0\right)$ satisfy condition (7). Let $\rho$ be the lifetime of the device. We have the following.

(a) Assume that $a_{2}$ is a convex function with $a_{2}(0)=0, X_{i}$ are DRHR and $Y$ is IFR. Then $\rho$ is IFR.

(b) Assume that $a_{2}$ is a concave function, $X_{i}$ are IFR and $Y$ is DRHR. Then $\rho$ is DRHR.

Proof Part (a) follows by Proposition 3.1(a). First of all, note that $\left(X^{*}(t), t \geq 0\right)$ is in the $\mathcal{I P I I}$ class. Actually, for a non-homogeneous Poisson process, $N^{*}(t)=N\left(a_{2}(t)\right)$, in which $(N(t), t \geq 0)$ is a standard Poisson process. Thus, $X(t)=\sum_{i=1}^{N(t)} X_{i}$ is a compound Poisson process and therefore it is in the $\mathcal{I P S I}$ class, as mentioned in Remark 2.8. As $X^{*}(t)=$ $X\left(a_{2}(t)\right)$, the assertion follows by Lemma 3.3(a). Secondly we will prove that $X^{*}(t) \uparrow_{\text {rh }}$. This follows as, if $s<t$, then $N\left(a_{2}(s)\right) \leq_{\operatorname{lr}} N\left(a_{2}(t)\right)(c f$. [16, p.62]) and, by this property we deduce for $X_{1}$ being DRHR

$$
\sum_{i=1}^{N\left(a_{2}(s)\right)} X_{i} \leq_{\mathrm{rh}} \sum_{i=1}^{N\left(a_{2}(t)\right)} X_{i}
$$

(see [17, Thm. 1.C.12]). Hence, the hypotheses in Proposition 3.1(a) are satisfied. Proof of part (b) is similar, using Proposition 3.1(b), taking into account Lemma 3.3(b) and again [17, Thm. 1.C.12]).

Remark 3.5 As mentioned before, Abdel-Hameed gave general conditions for a Lévy process in order to preserve the IFR property (see [12, Thm. 2.3(i)]). In particular for a compound Poisson process these conditions require that $X_{1}$ be log-concave (as the Lévy measure in the compound Poisson process is proportional to the distribution of $X_{1}$ ). Thus, in this case, Proposition 3.4(b) gives more general assumptions, under the requirement of $X_{1}$ to be DRHR. Note that the class DRHR contains, in particular, both log-concave and log-convex distributions. In fact, $X_{1}$ being log-concave implies that $X_{1}$ is both IFR and DRHR (recall Remark 2.4), so that, for a homogeneous Poisson process, this is a sufficient condition for the preservation of both the IFR and the DRHR property.

The next result provides preservation properties for the modified process $\left(X^{*}(t), t \geq 0\right)$ when the random variables in the process satisfy appropriate ageing properties. This, in particular, will allow us to deal with non-homogeneous gamma deterioration processes with trend.

Proposition 3.6 Let $(X(t), t \geq 0)$ be a process in the $\mathcal{I P S I}$ class. Consider a wear process in which $\left(X^{*}(t), t \geq 0\right)$ is as in (8). Assume that $Y$, the random threshold and $\left(X^{*}(t), t \geq 0\right)$ satisfy condition (7). Let $\rho$ be the lifetime of the device. We have the following.

(a) Assume that $X(t)$ are DRHR for all t and $X(0)=0$. Further, assume that $a_{1}$ and $a_{2}$ are increasing and convex functions, with $a_{1}(0)=a_{2}(0)=0$ and $Y$ is IFR. Then $\rho$ is IFR.

(b) Assume that $X(t)$ are IFR for all $t, a_{i}, i=1,2$ are increasing and concave functions and $Y$ is DRHR. Then $\rho$ is DRHR. 
Proof Part (a) will follow using Proposition 3.1(a). First of all, $\left(X^{*}(t), t \geq 0\right)$ is in the $\mathcal{I P I I}$ class due to Lemma 3.3(a). To show that $X^{*}(t) \uparrow_{\mathrm{rh}}$, note that, if $X(t)$ has the DRHR property, then $X(t) \uparrow_{\mathrm{rh}}$ (this is an immediate application of [17, Lem. 1.B.44] and the property of stationary and independent increments for $(X(t), t \geq 0))$. Then we have for $s<t$

$$
a_{1}(s)+X\left(a_{2}(s)\right) \leq_{\mathrm{rh}} a_{1}(t)+X\left(a_{2}(s)\right) \leq_{\mathrm{rh}} a_{1}(t)+X\left(a_{2}(t)\right) .
$$

The first inequality is obtained using Lemma [17, Lem. 1.B.44]) with $X:=a_{1}(s), Y:=a_{1}(t)$ and $Z:=X\left(a_{2}(s)\right)$, whereas the last inequality follows as the rh order is preserved by increasing transforms [17, Thm. 1.B.43]). Thus, the conditions in Proposition 3.1(a) follow, since $X^{*}(0)=0$, which proves part (a).

The proof of part (b) is very similar, using Proposition 3.1(b). Note that by Lemma 3.3(b) we find that $\left(X^{*}(t), t \geq 0\right)$ is in the $\mathcal{I P D I}$ class. Moreover, $X(t) \uparrow$ hr by [17, Lem. 1.B.3]. In this case, (12) holds if we replace the rh order by the hr order, using in this case [17, Lem. 1.B.3] and [17, Lem. 1.B.2].

Corollary 3.7 Let $(S(t), t \geq 0)$ be a gamma wear process. Consider a wear process in which $\left(S^{*}(t), t \geq 0\right)$ is as in (8). Let $\rho$ be the lifetime of the device.

(a) If $a_{1}$ and $a_{2}$ are increasing and convex functions, with $a_{1}(0)=a_{2}(0)=0$ and $Y$ is IFR, then $\rho$ is IFR.

(b) If $a_{1}(t)=0$ (no trend), $a_{2}$ is increasing and concave and $Y$ is DRHR, then $\rho$ is DRHR.

Proof Part (a) is an immediate application of Proposition 3.6(a). First of all note that a gamma process is in the $\mathcal{I P S I}$ class (recall Remark 2.8). Moreover, the random variables in $(S(t), t \geq 0)$ are absolutely continuous, so that condition (7) is satisfied. Finally, note that in a gamma process, the $S(t)$ are DRHR. This follows recalling Remark 2.4 as, if $t \leq 1, S(t)$ has log-convex density, whereas if $t \geq 1, S(t)$ has log-concave density (see [9, p.99]). Then the conditions in Proposition 3.6(a) hold and the result follows.

Part (b) follows as a consequence of Proposition 3.1(b). In fact, note that $S(t) \uparrow_{\mathrm{lr}}$ (see [16, p.62]), and this implies immediately that $S^{*}(t) \uparrow_{\mathrm{rr}}$. Thus, the conditions in Proposition 3.1(b) follow as, recalling Remark 2.6, $S^{*}(t) \uparrow_{\mathrm{hr}}$ and, using Lemma 3.3(b), $\left(S^{*}(t), t \geq 0\right)$ is in the $\mathcal{I P D I}$ class.

Remark 3.8 Abdel-Hameed [1] proved the IFR property for non-homogeneus gamma wear process, when the mean function is convex. Observe that the previous result extends this one, by adding a convex deterministic trend.

Remark 3.9 Note that, for the gamma process, we cannot proceed in a similar way to obtain a preservation result for the DRHR property, when we have a deterministic trend. In fact, if the IFR condition for $X(t)$ in Proposition 3.6(b) is not satisfied, we cannot ensure that $X^{*}(t) \uparrow \mathrm{hr}$. In fact, take $S^{*}(t)=t+S(t)$, a gamma process with linear trend. It is readily seen by calculus that $S^{*}(s) \not \mathbb{h r}_{\mathrm{hr}} S^{*}(t)$ if $0<s<t<1$ (the interval in which the IFR property fails).

Now, we focus on the DFR property. This property will follow immediately as a consequence of part (a) in Proposition 3.10 (part (b) will be used in the preservation of logconvex densities). The method of proof (with similar ideas to that in [19, Thm. 3.2]) differs 
substantially from the one used to obtain the preservation of the IFR property. In fact, for the DFR property we only need the stochastic ordering among the variables in the model, whereas for the IFR preservation property a stronger order (the rh one) was required in Proposition 3.1(b).

Proposition 3.10 Let $(X(t), t \geq 0)$ be a wear process. Assume that $(X(t), t \geq 0)$ is in the $\mathcal{I P D I}$ class. We have the following.

(a) Let $G:[0, \infty) \rightarrow[0, \infty)$ be a decreasing and log-convex function, with $G(x)>0, x \geq 0$ and right-continuous at $x=0$. Then $E G[X(t)]$ is a log-convex function on $[0, \infty)$.

(b) Let $G:(0, \infty) \rightarrow[0, \infty)$ be a decreasing and log-convex function, with $G(x)>0$, $x>0$. Assume that $P(X(t)=0)=0$ and that $E G[X(t)]<\infty$, for all $t>0$. Then $E G[X(t)]$ is a log-convex function on $(0, \infty)$.

Proof Let $I=[0, \infty)$ (case (a)) or $I=(0, \infty)$ (case (b)). Note that the log-convexity of $G$ on $I$ implies that $G$ is continuous on $I$. Thus, using conditions 1 and 4 in Definition 2.7 and similar arguments as in [15, Lem. 2.7], our conditions ensure the continuity of $T G(t):=$ $E G[X(t)]$ on $I$. Note that this last function is log-convex if and only if $h=\log T G(t)$ is convex. But using the fact (see [9, Exer. 3, p.73]) that for a continuous function $h$ we have

$$
h \text { is convex } \Leftrightarrow h\left(\frac{s+t}{2}\right) \leq \frac{h(s)+h(t)}{2}, \quad s<t,
$$

then we see, using $h=\log T G(t)$ and taking anti-logarithms, that

$$
T G(t) \text { is log-convex } \Leftrightarrow T G\left(\frac{s+t}{2}\right) \leq T G(s)^{1 / 2} T G(t)^{1 / 2}, \quad s<t .
$$

On the other hand, let $s<t$. Define $U:=X((s+t) / 2)-X(s)$ and $V:=X(t)-X((s+t) / 2)$. Using the property of independent increments, we can write

$$
T G\left(\frac{s+t}{2}\right)=E[G(X(s)+U)]=\int_{0}^{\infty} E[G(z+U)] d F_{X(s)}(z)
$$

Call $G_{z}(\cdot):=\frac{G(z+\cdot)}{G(z)}, z \in I$. Note that $G_{z}$ is a decreasing and log-convex function on $[0, \infty)$, with $G_{z}(x)>0, x \geq 0$, due to assumptions on $G$. Using this property we can easily show that

$$
E[G(z+U)] \leq G^{1 / 2}(z) E^{1 / 2}[G(z+U+V)] .
$$

Indeed, as $G_{z}$ is log-convex and $G_{z}(0)=1$, we have $G_{z}(x+y) \geq G_{z}(x) G_{z}(y), x, y \geq 0$ (this follows as $G_{z}(x+y)$ is TP2 (see [9, p.696])). Using this property and the fact that $U$ and $V$ are independent, we can write

$$
E\left[G_{z}(U+V)\right] \geq E\left[G_{z}(U)\right] E\left[G_{z}(V)\right] \geq E^{2}\left[G_{z}(U)\right],
$$

the last inequality due to the fact that the process is in the $\mathcal{I P D I}$ class (and therefore $\left.V \leq_{\text {st }} U\right)$ and that $G_{z}$ is decreasing. From the previous inequality we deduce easily (15). 
Using (14), (15) and Cauchy-Schwarz inequality, we obtain

$$
\begin{aligned}
T G\left(\frac{s+t}{2}\right) & \leq \int_{0}^{\infty} G^{1 / 2}(z) E^{1 / 2}[G(z+U+V)] d F_{X(s)}(z) \\
& \leq E^{1 / 2}[G(X(s))] E^{1 / 2}[G(X(t))]=T G^{1 / 2}(s) T G^{1 / 2}(t) .
\end{aligned}
$$

Thus, from (13) we deduce the log-convexity of $T G(t)=E G(X(t))$.

As an immediate consequence of part (a) in the previous result, we have the following.

Corollary 3.11 Let $(X(t), t \geq 0)$ be a process in the IPSI class. Consider a wear process in which $\left(X^{*}(t), t \geq 0\right)$ is as in (8), with $a_{1}$ and $a_{2}$ being increasing and concave functions. Assume that $Y$, the random threshold and $\left(X^{*}(t), t \geq 0\right)$ satisfy condition (7). Let $\rho$ be the lifetime of the device. If $Y$ is DFR, then $\rho$ is DFR.

Proof The result is immediate by Proposition 3.10. First of all, our conditions ensure that $\left(X^{*}(t), t \geq 0\right)$ is in the $\mathcal{I P D I}$ class, due to Lemma 3.3(b). Secondly, due to (1), we have $\bar{F}_{\rho}(t)=E\left[\bar{F}_{Y}\left(X^{*}(t)\right)\right]$. As $Y$ is DFR, then $G:=\bar{F}_{Y}$ satisfies assumptions on Proposition 3.10(a), from which we deduce the log-convexity of $\bar{F}_{\rho}$.

Remark 3.12 Observe that this result generalizes Theorem 2.3(iii) and Theorem 2.5 in [12], as we are able to add a deterministic trend.

\section{Preservation of log-concave and log-convex classes for subordinators}

The previous approach allows us to show the preservation of log-concave and log-convex classes under certain assumptions of the wear process $(X(t), t \geq 0)$. First of all, assume that this process is a subordinator (recall Remark 2.8) and that the process is also centered (that is, $E[X(t)]=t)$. In this case, it is well known $(c f .[20$, p.776]) that the Laplace transform of $(X(t), t \geq 0)$ can be written as

$$
E e^{-u X(t)}=e^{-t u \psi(u)}, \quad \psi(u):=E e^{-u U T}, \quad u \geq 0,
$$

where $U$ and $T$ are independent random variables such that $U$ is uniformly distributed on $[0,1]$ and $T$ is non-negative. The random variable $T$, which determines the process, will be called the characteristic random variable of $(X(t), t \geq 0)$. Using this fact, if we consider $U$ and $T$ as before, being independent of $(X(t), t \geq 0)$, for any absolutely continuous random variable $Y$, we have the following identity (cf. [20, Prop. 4]):

$$
\frac{d}{d t} E F_{Y}(X(t))=E f_{Y}(X(t)+U T), \quad t>0,
$$

provided that the expectation on the right-hand side is finite. Observe that the process $\left(X_{1}(t), t \geq 0\right)$, where $X_{1}(t)=X(t)+U T$, belongs to the $\mathcal{I P S I}$ class. Therefore, as an immediate consequence of (1) and Proposition 3.10(b), the log-convexity of $\rho$ is guaranteed, provided that $Y$ has the analogous property. Moreover, if we transform a centered subordinator $(X(t), t \geq 0)$ into a wear process $X^{*}(t):=X\left(a_{2}(t)\right)$, in which $a_{2}(t)$ is an increasing and differentiable function, the chain rule allows us to write

$$
\frac{d}{d t} E F_{Y}\left(X^{*}(t)\right)=a_{2}^{\prime}(t) E f_{Y}\left(X\left(a_{2}(t)\right)+U T\right), \quad t>0 .
$$


Note firstly that, for results concerning log-concavity or log-convexity we will always assume that $a_{2}(0)=0$, in order to guarantee that $\rho$ does not have positive mass at 0 , and therefore it is an absolutely continuous random variable. In fact, note that under this assumption, we have by (1), and by the fact that $X(0)=0$ (as the process is a centered subordinator), $P(\rho=0)=E F_{Y}(X(0))=F_{Y}(0)=0$.

With respect to log-convexity, we have the following result.

Proposition 4.1 Let $(X(t), t \geq 0)$ be a centered subordinator. Consider a wear process in which $\left(X^{*}(t), t \geq 0\right)$ is defined as $X^{*}(t)=X\left(a_{2}(t)\right)$, and let $Y$ be the random threshold. Let $\rho$ be the lifetime of the device. If $Y$ is log-convex and $a_{2}$ is differentiable, with $a_{2}(0)=0, a_{2}^{\prime}$ being non-negative, decreasing, and log-convex, then $\rho$ is log-convex.

Proof Applying (1) and (18), we have

$$
\frac{d}{d t} F_{\rho}(t)=\frac{d}{d t} E F_{Y}\left(X^{*}(t)\right)=a_{2}^{\prime}(t) E f_{Y}\left(X\left(a_{2}(t)\right)+U T\right) .
$$

The conditions about $a_{2}$ guarantee that this function is concave, and therefore by Lemma 3.3(b), the process $\left(X\left(a_{2}(t)\right)+U T, t \geq 0\right)$ is in the $\mathcal{I P D I}$ class. As $Y$ is log-convex, then $f_{Y}$ is log-convex, decreasing, and strictly positive on $(0, \infty)$ (see [9, Prop. C.11, p.117]). Thus, we can apply Proposition 3.10(b), so that the second factor in (19) is a log-convex function, and the result follows for the log-convexity of $a_{2}^{\prime}$, as the product of log-convex functions is log-convex.

Remark 4.2 The previous result guarantees, obviously, the preservation of the logconvexity for a process in the $\mathcal{I P S I}$ class $\left(X^{*}(t), t \geq 0\right)$ in which $E\left[X^{*}(t)\right]=\lambda t$, as $(X(t):=$ $\left.X^{*}(t / \lambda), t \geq 0\right)$ is a centered subordinator and in this case $a_{2}(t)=\lambda t$. A non-trivial example of a function $a_{2}$ satisfying the hypotheses in the previous results is such that $a_{2}(t)=\int_{0}^{t} e^{-u} u^{\alpha-1} d u, t>0$, with $0<\alpha \leq 1$.

For the preservation of log-concavity, stronger assumptions, concerning stochastic ordering properties of the derived process, are needed. For this reason we present specific examples in which these properties can be checked. First of all, we present a log-concavity result for the compound Poisson process.

Proposition 4.3 Let $\left(X^{*}(t), t \geq 0\right)$ be a compound Poisson process, that is,

$$
X^{*}(t)=\sum_{i=1}^{N^{*}(t)} X_{i}
$$

in which $\left(N^{*}(t), t \geq 0\right)$ is a homogeneous Poisson process, and $\left(X_{n}\right)_{n=1,2, \ldots}$ is a sequence of independent, identically distributed non-negative random variables, having finite mean and being independent of the process. Let $Y$ be a log-concave random threshold and let $\rho$ be the lifetime of the device. If $X_{1}$ is log-concave, then $\rho$ is log-concave.

Proof Let $\mu:=E\left[X_{1}\right]$ and let $(N(t), t>0)$ be a Poisson process of rate $1 / \mu$. Then $X(t):=$ $\sum_{i=1}^{N(t)} X_{i}$ is a centered subordinator, and therefore (16) holds true. Let $\psi_{X_{1}}(u):=E e^{-u X_{1}}$ be 
the Laplace transform of $X_{1}$. Taking into account that the Laplace transform of $X(t)$ is given by $E e^{-u X(t)}=\exp \left(-(t / \mu)\left(1-\psi_{X_{1}}(u)\right)\right)(c f .[21, \mathrm{p} .84])$, we obtain

$$
\frac{1-\psi_{X_{1}}(u)}{\mu}=u \psi_{U T}(u) \Rightarrow \psi_{U T}(u)=\frac{1-\psi_{X_{1}}(u)}{\mu u} .
$$

Let $X^{e}$ be a random variable having the equilibrium distribution of $X_{1}(c f .[9, \mathrm{p} .18])$, that is, $f_{X^{e}}(x)=\bar{F}_{X_{1}}(x) / \mu$. It is readily seen using integration by parts that $\psi_{X_{e}}(u)=(\mu u)^{-1}(1-$ $\left.\psi_{X_{1}}(u)\right)$, and therefore $U T={ }_{\text {st }} X_{e}$. As we have $X^{*}(t)=$ st $X(\mu \lambda t)$, in which $\lambda$ is the rate of $\left(N^{*}(t), t \geq 0\right)$, we have, using (18),

$$
\frac{d}{d t} E\left[F_{Y}\left(X^{*}(t)\right)\right]=\mu \lambda E f_{Y}\left(\sum_{i=1}^{N(\mu \lambda t)} X_{i}+X^{e}\right), \quad t>0 .
$$

The process $\left(\sum_{i=1}^{N(\mu \lambda t)} X_{i}+X^{e}, t \geq 0\right)$ is in the $\mathcal{I} \mathcal{P} \mathcal{I}$ class. Moreover, as $X_{1}$ is log-concave, $\sum_{i=1}^{N(\mu \lambda t)} X_{i} \uparrow_{\mathrm{lr}}$ [17, Thm. 1.C.11]. On the other hand, if $X_{1}$ is log-concave, then it is IFR, and, therefore, $X^{e}$ is log-concave. Thus, $\sum_{i=1}^{N(\mu \lambda t)} X_{i}+X^{e} \uparrow_{\text {Ir }}$ [17, p. 47], and by Theorem 2.9(c), the expression in (20) is a log-concave function, so that the conclusion holds.

We consider now a partial result for the gamma process. It is well known that, in this case, $T$ is an exponential random variable (cf. [22, p.957]). On the other hand, the density of $U T$, using the product distribution formula, can be written as

$$
f_{U T}(x)=\int_{0}^{1} \frac{1}{u} e^{-x / u} d u=\int_{1}^{\infty} v^{-1} e^{-x v} d v .
$$

Note that the previous expression shows us that $f_{U T}$ is completely monotone, and henceforth log-convex [21, p.123]. Thus, $U T$ is log-convex. The log-convexity of this random variable allows us to give the following result, under the assumption of a log-concave decreasing density of $Y$.

Proposition 4.4 Let $(S(t), t \geq 0)$ be a gamma wear process. Consider a wear process in which $\left(S^{*}(t)=S\left(a_{2}(t)\right), t \geq 0\right)$. Let $Y$ be the random threshold, and let $\rho$ be the lifetime of the device. If $Y$ has a log-concave and decreasing density, $a_{2}$ is differentiable, with $a_{2}(0)=0$, $a_{2}^{\prime}$ being non-negative, increasing, and log-concave, then $\rho$ is log-concave.

Proof The derived process for $(S(t), t \geq 0)$ in this case is $\left(S_{1}(t):=S(t)+U T, t \geq 0\right)$, in which the density of $U T$ is given in (21). Therefore, by (18), we obtain

$$
\frac{d}{d t} E\left[F_{Y}\left(S^{*}(t)\right)\right]=a_{2}^{\prime}(t) E f_{Y}\left(S\left(a_{2}(t)\right)+U T\right), \quad t>0 .
$$

Our conditions guarantee that $a_{2}$ is convex, and thus $\left(S\left(a_{2}(t)\right)+U T, t \geq 0\right)$ is in the $\mathcal{I P I I}$ class, thanks to Lemma 3.3(a). Moreover, $S\left(a_{2}(t)\right)+U T$ is DRHR for all $t \geq 0$. This follows as $U T$ has a log-convex density, and therefore it is DRHR (recall Remark 2.6). Moreover, $S\left(a_{2}(t)\right)$ is always DRHR (as its density is either log-concave or log-convex). Thus, the DRHR property for $S\left(a_{2}(t)\right)+U T$ follows, as this property is closed under convolution [9, p.179]. Then it follows easily [17, Lem. 1.B.44]) that $S\left(a_{2}(t)\right)+U T \uparrow_{\mathrm{rh}}$. Thus, the conclusion follows by (22) and Theorem 2.9(a). 
Remark 4.5 The conditions in the previous result are quite restrictive. However, the random threshold $Y$ will have a log-concave decreasing density if it has an exponential distribution, or a uniform distribution on the interval $(0, a)$, for some $a>0$. On the other hand, the function $a_{2}(t)=e^{c t}-1, t \geq 0$ or $a_{2}(t)=c t, t \geq 0$ for $c>0$ verifies the conditions in Proposition 4.4.

If the random variables in the derived process were ordered in the likelihood ratio order, then we could generalize the previous result, by using Theorem 2.9(c). The technical problem is the complexity of the density of $S(t)+U T$ (we can find integral expressions, but we are not able to find a closed-form expression). As a partial result we are able to check the preservation of log-concavity on the interval $[1, \infty)$. The next lemma will be very useful to this end.

Lemma 4.6 The random variable $S(1)+U T$, in which $S(1)$ and $T$ are exponential random variables with mean 1 and $U$ is uniform (all of them independent), is log-concave.

Proof Let $\left(N_{i}(t), t \geq 0\right), i=1,2$ be two independent standard Poisson processes. Applying [21, Thm. 6.13, p.347], to check the log-concavity of $S(1)+U T$ one can check equivalently the discrete log-concavity of $N_{1}(\theta(S(1)+U T))$, for all $\theta>0$. The property of independent and stationary increments for the Poisson processes implies that

$$
N_{1}(\theta(S(1)+U T))={ }_{\text {st }} N_{1}(\theta S(1))+N_{2}(\theta U T) .
$$

The first term has a geometric distribution with parameter $p:=\theta(\theta+1)^{-1}($ see $[21, \mathrm{p} .372]$, for instance), that is,

$$
P\left(N_{1}(\theta S(1))=k\right)=\frac{\theta^{k}}{(\theta+1)^{k+1}}, \quad k=0,1, \ldots
$$

As the second summand in (23) is in distribution like the first one, replacing the parameter $\theta$ by the random variable $\theta U$, we have

$$
p_{k}:=P\left(N_{2}(\theta U T)=k\right)=\int_{0}^{1} \frac{(u \theta)^{k}}{(u \theta+1)^{k+1}} d u, \quad k=0,1, \ldots
$$

Thus in (23) we have the convolution of a geometric random variable with a random variable having probability mass function as given above. In [23, Lem. 4.3] it was shown that a sufficient condition for a geometric convolution like in (23) to be log-concave is that $p_{k+1} p_{k}^{-1} \leq \theta(\theta+1)^{-1}, k=1,2, \ldots$ But taking into account that $u \theta(u \theta+1)^{-1} \leq \theta(\theta+1)^{-1}$, for all $0 \leq u \leq 1$, we have

$$
\frac{p_{k+1}}{p_{k}}=\frac{\int_{0}^{1} \frac{(u \theta)^{k+1}}{(u \theta+1)^{k+2}} d u}{\int_{0}^{1} \frac{(u \theta)^{k}}{(u \theta+1)^{k+1}} d u} \leq \frac{\theta}{\theta+1}, \quad k=1,2, \ldots
$$

thus showing the discrete log-concavity for $N_{1}(\theta(S(1)+U T))$ and, therefore, the logconcavity for $S(1)+U T$. 
Proposition 4.7 Let $(S(t), t \geq 0)$ be a gamma wear process. Let $Y$ be the random threshold, and let $\rho$ be the lifetime of the device. If $Y$ has a log-concave density, then $\rho$ is log-concave on $[1, \infty)$.

Proof Consider the stochastic process $\left(S^{*}(t), t \geq 0\right)$, in which $S^{*}(t)=S(t+1)$. Using (18), we obtain

$$
\frac{d}{d t} E\left[F_{Y}(S(t))\right]=E f_{Y}(S(t)+U T)=E f_{Y}\left(S^{*}(t-1)+U T\right), \quad t \geq 1 .
$$

Consider now the stochastic process $S_{1}^{*}(t):=S(t+1)+U T, t \geq 0$. Note that $S_{1}^{*}(t) \uparrow_{\mathrm{lr}}$. This follows as $S_{1}^{*}(t)=$ st $S(1)+U T+S^{\prime}(t)$, in which $S^{\prime}(t)$ is a gamma random variable of shape parameter $t$ independent of $S(1)+U T$. Thus, for $0 \leq t_{1}<t_{2}$, we have $S^{\prime}\left(t_{1}\right) \leq_{\operatorname{lr}} S^{\prime}\left(t_{2}\right)$ As by Lemma 4.6 $S(1)+U T$ is log-concave, we have $S(1)+U T+S^{\prime}\left(t_{1}\right) \leq_{\operatorname{lr}} S(1)+U T+S^{\prime}\left(t_{2}\right)$ (see $[17, \mathrm{p} .46])$, thus proving the likelihood ratio order assumption. Then, by Theorem $2.9(\mathrm{c})$ $f(t):=E f_{Y}\left(S^{*}(t)+U T\right), t>0$, is a log-concave function, and so is (26), as if $f(t), t>0$ is a $\log$-concave function on $(0, \infty)$, then $g(t):=f(t-1), t>1$ is a log-concave function on $(1, \infty)$. Then the conclusion follows by the log-concavity of (26) and (1).

Remark 4.8 If we could extend the fact that $S(t)+U T \uparrow_{\text {Ir }}$ from $t \geq 1$ to $t \geq 0$, then we could prove the preservation of log-concavity on $(0, \infty)$. However, due to the technical complexity of the density function $S(t)+U T$ we are not able, at this point, to prove or disprove this fact.

\section{Competing interests}

The authors declare that they have no competing interests.

\section{Authors' contributions}

All authors read and approved the final manuscript.

\section{Author details}

'Department of Statistical Methods, University of Zaragoza, Zaragoza, 50009, Spain. ${ }^{2}$ Department of Statistical Methods, University of Zaragoza, Zaragoza, 50018, Spain. ${ }^{3}$ Department of Statistics, Ewha Womans University, Seoul, 120-750, Korea.

\section{Acknowledgements}

This work has been supported by the Spanish research project MTM2012-36603-C02-02. The first and second authors acknowledge the support of DGA S11 and E64, respectively. The work of the third author was supported by the National Research Foundation of Korea (NRF) grant funded by the Korea government (MEST) (No. 2011-0017338). The work of the third author was also supported by Priority Research Centers Program through the National Research Foundation of Korea (NRF) funded by the Ministry of Education, Science and Technology (2009-0093827).

Received: 21 February 2014 Accepted: 5 May 2014 Published: 20 May 2014

\section{References}

1. Abdel-Hameed, M: A gamma wear process. IEEE Trans. Reliab. 24, 152-153 (1975)

2. Nicolai, RP, Frenk, JBG, Dekker, R: Modeling and optimizing imperfect maintenance of coatings on steel structures. Struct. Saf. 31, 234-244 (2009)

3. van Noortwijk, JM: A survey of the application of gamma processes in maintenance. Reliab. Eng. Syst. Saf. 94, 2-21 (2009)

4. Abdel-Hameed, M, Proschan, F: Shock models with underlying birth process. J. Appl. Probab. 12, 18-28 (1975)

5. Park, C, Padgett, WJ: Accelerated degradation models for failure based on geometric Brownian motion and gamma processes. Lifetime Data Anal. 11, 511-527 (2005)

6. Paroissin, C, Rabehasaina, L: First and last passage times of spectrally positive Lévy processes with application to reliability. Methodol. Comput. Appl. Probab. (in press). doi:10.1007/s11009-013-9360-9

7. Belzunce, F, Ortega, E, Ruiz, JM: On ageing properties of first passage times of increasing Markov processes. Adv. Appl. Probab. 34, 241-259 (2002)

8. Abdel-Hameed, M: Life distribution of devices subject to a pure-jump damage process. J. Appl. Probab. 21, 816-825 (1984) 
9. Marshall, AW, Olkin, I: Life Distributions. Springer, New York (2007)

10. Shaked, M, Shanthikumar, G: On the first-passage times of pure jump processes. J. Appl. Probab. 25, 501-509 (1988)

11. Esary, JD, Marshall, W, Proschan, F: Shock models and wear processes. Ann. Probab. 4, 627-649 (1973)

12. Abdel-Hameed, M: Life distribution of devices subject to Lévy wear process. Math. Oper. Res. 9, 606-614 (1984)

13. Abdel-Hameed, M: Degradation processes: an overview. In: Advances in Degradation Modeling, pp. 17-25. Birkhäuser, Boston (2010)

14. Badía, FG: On the preservation of log-convexity and log-concavity under some classical Bernstein-type operators. J. Math. Anal. Appl. 360, 485-490 (2009)

15. Badía, FG, Sangüesa, C: Log-concavity for Bernstein-type operators using stochastic orders. J. Math. Anal. Appl. 413, 953-962 (2014)

16. Müller, A, Stoyan, D: Comparison Methods for Stochastic Models and Risks. Wiley, Chichester (2002)

17. Shaked, M, Shanthikumar, JG: Stochastic Orders. Springer, New York (2007)

18. Sato, Kl: Lévy Processes and Infinitely Divisible Distributions. Cambridge University Press, Cambridge (1999)

19. Badía, FG, Sangüesa, C: Preservation of reliability classes under mixtures of renewal processes. Probab. Eng. Inf. Sci. 22 1-17 (2008)

20. Adell, JA, Lekuona, A: Taylor's formula and preservation of generalized convexity for positive linear operators. J. Appl. Probab. 37, 765-777 (2000)

21. Steutel, F, van Harn, K: Infinite Divisibility of Probability Distributions on the Real Line. Dekker, New York (2004)

22. Adell, JA: Asymptotic estimates for Stieltjes constants: a probabilistic approach. Proc. R. Soc. Lond., Ser. A, Math. Phys. Eng. Sci. 467, 954-963 (2011)

23. Johnson, O, Goldschmidt, C: Preservation of log-concavity on summation. ESAIM Probab. Stat. 10, 206-215 (2006)

10.1186/1029-242X-2014-200

Cite this article as: Sangüesa et al.: Preservation of ageing classes in deterioration models with independent increments. Journal of Inequalities and Applications 2014, 2014:200

\section{Submit your manuscript to a SpringerOpen ${ }^{\circ}$ journal and benefit from:}

- Convenient online submission

- Rigorous peer review

- Immediate publication on acceptance

- Open access: articles freely available online

- High visibility within the field

- Retaining the copyright to your article 